\title{
ON POSITIVITY OF FOURIER TRANSFORMS
}

\author{
E.O. TUCK
}

This note concerns Fourier transforms on the real positive line. In particular, we seek conditions on a real function $u(x)$ in $x>0$, that ensure that its Fourier-cosine transform $v(t)=\int_{0}^{\infty} u(x) \cos x t d x$ is positive. We prove first that this is so for all $t>0$, if $u^{\prime \prime}(x)>0$ for all $x>0$, that is, that everywhere-convex functions have everywhere-positive Fourier-cosine transforms. We then obtain a complex-plane criterion for some types of non-convex $u(x)$. Finally we consider criteria on $u(x)$ that imply positivity of $v(t)$ for $t>t_{0}$, for some $t_{0}>0$.

\section{INTRODUCTION}

Define for $t>0$ the ordinary Fourier-cosine transform

$$
v(t)=\int_{0}^{\infty} u(x) \cos x t d x
$$

with inverse

$$
u(x)=\frac{2}{\pi} \int_{0}^{\infty} v(t) \cos x t d t
$$

with a similar definition for the Fourier-sine transform.

Generally we shall assume here that $u(x)$ is real and smooth in $x>0$ and that the Fourier integral (1) converges. In particular, $u(x)$ and all of its derivatives are bounded everywhere in $x>0$ and tend to zero as $x \rightarrow+\infty$. Meanwhile, $u(x)$ could be bounded at the origin, but more generally could have a weak singularity, with $x u(x) \rightarrow 0$ as $x \rightarrow 0_{+}$, that is, $u(x)$ grows at a rate less than $x^{-1}$. For Fourier-sine transforms, we can allow a stronger singularity at $x=0_{+}$, with any growth rate less than $x^{-2}$. We shall also generalise the results later, to allow even stronger singularities at the origin.

One important class of functions $u(x)$ is "convex", that is, such that $u^{\prime \prime}(x)>0$ for all $x>0$, which implies (since $u^{\prime}(+\infty)=0$ ) that $u^{\prime}(x)<0$ and (since $u(+\infty)=0$ ) that $u(x)>0$. That is, convex functions possessing Fourier transforms are also decreasing and positive. Such convex functions need not be smooth at $x=0$, indeed not even bounded so long as they are integrable. In particular, they need not (indeed cannot) have all of their

Received 29th March, 2006

Copyright Clearance Centre, Inc. Serial-fee code: 0004-9727/06 \$A2.00+0.00. 
odd-order derivatives zero at $x=0_{+}$, and hence do not extend smoothly as even functions into $x<0$. We shall show that convex functions have everywhere-positive Fourier-cosine transforms. An elementary convex example is $u(x)=e^{-x}$ with $v(t)=1 /\left(1+t^{2}\right)>0$.

However, we are more interested here in non-convex functions $u(x)$ which are bounded, positive and decreasing in $x>0$, which extend smoothly as an even function to the whole real line, that is, all odd-order derivatives vanish at $x=0_{+}$, and which usually have a single inflexion point in $x>0$. Let us call such functions "bell-shaped" functions.

Some bell-shaped functions have positive Fourier transforms, and some don't. Thus compare $u(x)=1 /\left(1+x^{2}\right)$, which has transform $v(t)=(\pi / 2) e^{-t}$, with $u(x)=1 /(1$ $\left.+x^{4} / 4\right)$, which has transform $v(t)=(\pi / 2) e^{-t}(\cos t+\sin t)$. One $v(t)$ is positive, the other oscillates between positive and negative values, but both $u(x)$ are bell-shaped and have quite similar graphs. A criterion for discriminating between such bell-shaped functions would be of some value.

\section{PROOF OF POSITIVITY FOR CONVEX FUNCTIONS}

Positivity of Fourier-sine transforms is somewhat easier to prove than that of Fouriercosine transforms. But by integration by parts we have

$$
v(t)=-\frac{1}{t} \int_{0}^{\infty} u^{\prime}(x) \sin x t d x
$$

given that the assumed convergence requirements $(u \rightarrow 0$ as $x \rightarrow+\infty$ and $x u(x) \rightarrow 0$ as $x \rightarrow 0_{+}$) eliminate the integrated part. That is, the Fourier-cosine transform of $u(x)$ is $-1 / t$ times the Fourier-sine transform of its derivative $u^{\prime}(x)$.

Now let us prove that the Fourier-sine transform of a decreasing function $w(x)$ is positive. That is,

$$
\begin{aligned}
\int_{0}^{\infty} w(x) \sin x t d x & =\sum_{j=0}^{\infty} \int_{2 \pi j / y}^{2 \pi(j+1) / y} w(x) \sin x t d x \\
& =\frac{1}{t} \sum_{j=0}^{\infty} \int_{0}^{2 \pi} w\left(\frac{2 \pi j+\theta}{t}\right) \sin \theta d \theta \\
& =\frac{1}{t} \sum_{j=0}^{\infty} \int_{0}^{\pi}\left[w\left(\frac{2 \pi j+\theta}{t}\right)-w\left(\frac{2 \pi j+\theta}{t}+\frac{\pi}{t}\right)\right] \sin \theta d \theta
\end{aligned}
$$

If $w(x)$ is a decreasing function for all $x$, the quantity in square brackets is positive for all $t$ and all $j$, and so is $\sin \theta$ in $(0, \pi)$; hence the Fourier-sine transform is positive. This is essentially a simple geometrical result, each negative half-period loop of the sine function contributing less to the sum than the positive half-period loop preceeding it. 
Now define $w(x)=-u^{\prime}(x)$. Then $u^{\prime \prime}(x)>0$ implies $w^{\prime}(x)<0$ so this $w(x)$ is a decreasing function. Therefore its Fourier-sine transform is positive, and hence so is the Fourier-cosine transform of $u(x)$. Thus we have proved that $u^{\prime \prime}(x)>0$ for all $x>0$ guarantees $v(t)>0$ for all $t>0$. That is, convex functions have everywhere-positive Fourier-cosine transforms.

However, bell-shaped functions are not convex, and it is doubtful if there is any criterion based solely on behaviour of $u(x)$ for positive real $x$, for positivity of the Fouriercosine transform of bell-shaped functions. Somewhat reluctantly, we must move into the complex plane.

\section{COMPLEX DETOURS}

Suppose we can continue the function $u(z)$ into the upper half complex $z$ plane, and that it is an even analytic function of $z$, real on the real axis, satisfying $\bar{u}(z)=u(\bar{z})$. Then we can write

$$
v(t)=\frac{1}{2} \int_{-\infty}^{\infty} u(z) e^{i z t} d z
$$

Now suppose that $|u(z)| \rightarrow 0$ as $\Re z \rightarrow \pm \infty$ for some range of positive values of the imaginary part of $z$, say for $\Im z<p$. Then we can shift the path of integration upward, writing $z=x+i p$ and giving

$$
\begin{aligned}
v(t) & =\frac{1}{2} e^{-p t} \int_{-\infty}^{\infty} u(x+i p) e^{i x t} d x \\
& =e^{-p t} \int_{0}^{\infty}[\Re u(x+i p) \cos x t-\Im u(x+i p) \sin x t] d x .
\end{aligned}
$$

Equation (7) expresses $v(t)$ as the sum of a Fourier cosine and a Fourier sine transform, each multiplied by an exponential decay factor. Hence if $\Re u(x+i p)$ is convex (and decreasing and positive) and also $-\Im u(x+i p)$ is decreasing (and positive), then $v(t)$ is positive for all $t>0$.

An example is $u(z)=1 / \sqrt{1+z^{2}}$ where we can take $p=1$. Then $\Re u(x+i)=R \cos \theta$ and $-\Im u(x+i)=R \sin \theta$, where $R=x^{-1 / 2}\left(x^{2}+4\right)^{-1 / 4}$ and $\tan 2 \theta=2 / x$. These functions have the required properties, which proves that $v(t)$ is positive for all $t>0$. In fact, $v(t)=K_{0}(t)$ is a modified Bessel function [1], which is indeed positive and decays exponentially for large $t$.

\section{NON-INTEGRABLE SINGULARITIES}

The above analysis is valid as it stands if $u(z)$ is integrable along the whole line $z=x+i p$, including the case of bounded $u(z)$. However, it is of no use for the present purpose if $u(z)$ is bounded as $z \rightarrow i p$, because then evenness of $u(z)$ necessarily implies 
that $\Im u(i p)=0$, so $-\Im u(x+i p)$ cannot be decreasing and positive for $x>0$. Thus we are only interested in choices of $p$ such that $u(z)$ has a singularity at $z=i p$ on the imaginary axis, and no other singularity closer to the origin. The above example $u(z)=1 / \sqrt{1+z^{2}}$ has an (integrable) inverse square root branch point at $z=i$.

But what if the nearest singularity is stronger than that? For example, $u(z)$ $=1 /\left(1+z^{2}\right)$ is not integrable through the simple pole at $z=i$, nor is $u(z)=\left(1+z^{2}\right)^{-\alpha}$ for any $\alpha \geqslant 1$. Nevertheless these happen to be functions with positive Fourier-cosine transforms. We would like to be able to prove that statement using methods like those in the previous section. For the present, we shall only discuss the simple-pole case $\alpha=1$; although a similar analysis can be performed for stronger singularities, it requires generalisation of the concept of a Fourier transform to non-integrable functions.

Thus we now assume that as $z \rightarrow i p$ we have

$$
u(z) \rightarrow U_{0}[i(z-i p)]^{-1}
$$

for some real constant $U_{0}$. The example $u(z)=1 /\left(1+z^{2}\right)$ has $U_{0}=1 / 2$. Note that when (8) holds, only the imaginary part of $u$ is singular as $x \rightarrow 0_{+}$on the line $z=x+i p$, with $-x \Im u(x+i p) \rightarrow U_{0}$, but $x \Re u(x+i p) \rightarrow 0$. Hence both Fourier integrals in (7) converge in spite of the non-integrable character of the singularity in $u(z)$. Nevertheless we must modify (7) to take account of the pole.

The necessary modification is simply to allow the path of integration to pass below the pole, on a semicircle of vanishingly small radius. The net effect is to add a term proportional to the residue at the pole, so (7) becomes

$$
v(t)=e^{-p t}\left[\int_{0}^{\infty}[\Re u(x+i p) \cos x t-\Im u(x+i p) \sin x t d x]+U_{0} \frac{\pi}{2}\right] .
$$

For example, suppose $u(z)=1 /\left(1+z^{2}\right)$ and $p=1$. Then

$$
v(t)=e^{-t}\left[\int_{0}^{\infty} \frac{1}{x^{2}+4} \cos x t d x+\int_{0}^{\infty} \frac{2}{x\left(x^{2}+4\right)} \sin x t d x+\frac{\pi}{4}\right] .
$$

Since the coefficient of $\sin x t$ is positive and decreasing, the Fourier-sine integral in (10) is positive. Although the coefficient of $\cos x t$ is not convex, we no longer need the Fouriercosine integral to be positive (though it is!), so long as it is overwhelmed by the positive correction term $\pi / 4$. This is clearly so, since (replacing $\cos x t$ by -1 ), the Fourier-cosine integral can be seen to be greater than $-\pi / 4$. Hence $v(t)>0$. Of course, given that we can actually evaluate this $v(t)=(\pi / 2) e^{-t}$ and the other Fourier integrals in (10), this appears a clumsy way to prove something obvious, but is important in principle, in that it does not depend on a knowledge of the exact integrals, so generalises to more complicated functions. 


\section{POSITIVITY ONLY FOR $t>t_{0}$}

In fact, in some applications it is neither necessary nor desirable to insist that $v(t)>0$ for all $t>0$, and it may be enough to show that there is a finite $t_{0}>0$ such that $v(t)>0$ for all $t>t_{0}$. Can we find criteria on $u(x)$ for this to be true, and if so, can we estimate $t_{0}$ ? Only preliminary discussions of this generalised task are given here.

Assuming validity of (7), that is, ruling out for the time being non-integrable singularities, on integration of the first term of $(7)$ by parts, $v(t)$ can be expressed as a single Fourier-sine integral

$$
v(t)=e^{-p t} \int_{0}^{\infty} F(x ; t) \sin x t d x
$$

where

$$
\begin{aligned}
F(x ; t) & =-\Im u(x+i p)-\frac{1}{t} \frac{d}{d x} \Re u(x+i p) \\
& =\Re\left[i u(x+i p)-\frac{1}{t} u^{\prime}(x+i p)\right] .
\end{aligned}
$$

Now if (in any range of $t$ values) the function $F(x ; t)$ is a decreasing (and positive) function of $x$ for all $x>0$, then $v(t)$ is positive for that range of $t$. This is true for all $t$ when the two terms of (12) are both decreasing and positive for all $x>0$, as in the examples already given.

However, suppose it is not true for all $t$, but only for $t>t_{0}$, for some $t_{0}>0$. Then in particular it must be true for large $t$, when the second term of (12) tends to zero, so the first term $F(x ; \infty)=-\Im u(x+i p)$ of $(12)$ must be decreasing and positive for all $x>0$. If the second term was also decreasing and positive for all $x>0$, we would have $t_{0}=0$ as above, so let us assume that this is not so for some $x$ values. Then there is still a chance of finding a finite $t_{0}$ such that the sum of the two terms of (12) is decreasing and positive for all $x>0$. This will be possible if the second term of (12) is bounded (together with its derivative) in $x>0$, and does not become asymptotically large relative to the first term, either as $x \rightarrow 0_{+}$or as $x \rightarrow \infty$.

For example, consider

$$
\int_{0}^{\infty} \frac{\sin x t-x \cos x t}{1+x^{2}} d x=e^{-t} \operatorname{Ei}(t)
$$

where $E \mathrm{i}$ is the exponential integral $([1$, p. 230]). Now

$$
\int_{0}^{\infty} \frac{\sin x t-x \cos x t}{1+x^{2}} d x=\int_{0}^{\infty} F(x ; t) \sin x t d x
$$

where

$$
F(x ; t)=\frac{1}{1+x^{2}}+\frac{1}{t} \frac{1-x^{2}}{\left(1+x^{2}\right)^{2}}
$$


is positive and decreasing for all $x$ if $t>t_{0}=1$. This is a conservative estimate of $t_{0}$, since in fact $\operatorname{Ei}(t)>0$ for all $t>0.37253$.

There is a potential application to the celebrated Riemann hypothesis [2]. This hypothesis might well be true if $v(t)=V^{\prime}(t)^{2}-V(t) V^{\prime \prime}(t)$ could be proved positive for all $t>t_{0}$, where $V(t)=\pi^{-s / 2} \Gamma(s / 2) \zeta(s)$ is a real-valued scaling of the Riemann zeta function $([1$, p. 807]) on its critical line $s=1 / 2+i t$. Numerical evidence [4] is that this is so with $t_{0} \approx 5.9009$, but a proof is elusive. The inverse Fourier transform of this $v(t)$ is the bell-shaped function

$$
u(x)=\frac{1}{4} \int_{0}^{\infty} y^{2} U\left(\frac{x+y}{2}\right) U\left(\frac{x-y}{2}\right) d y,
$$

where

$$
U(x)=-2 e^{-x / 2}+4 e^{x / 2} \sum_{n=1}^{\infty} e^{-n^{2} \pi e^{2 x}}
$$

is the (also bell-shaped) inverse Fourier transform of the (sign-oscillatory) Riemann function $V(t)[3]$. The nearest singularity of $u(z)$ is at $z=i \pi / 2$, so we could try $p=\pi / 2$ in the above. However, there also appear to be many other singularities along the line $z=x+i \pi / 2$, which may or may not be integrable. Further study of $u(z)$ near that line would seem to be of value.

\section{REFERENCES}

[1] M. Abramowitz and I.A. Stegun, Handbook of mathematical functions, with formulas, graphs and mathematical tables (Dover Publications, New York, 1964).

[2] H.M. Edwards, Riemann's zeta function (Academic Press, New York, 1974).

[3] J.M. Hill, 'On some integrals involving functions $\phi(x)$ such that $\phi(1 / x)=\sqrt{x} \phi(x)$ ', J. Math. Anal. Appl. 309 (2005), 256-270.

[4] E.O. Tuck, 'When does the first derivative exceed the geometric mean of a function and its second derivative?', Austral. Math. Soc. Gaz. 32 (2005), 267-268.

Applied Mathematics

The University of Adelaide

Australia 5005 